for workers. Some culture-specific coping behaviors to combat work-related fatigue such as the use of betel nuts, alcoholic energy drinks and substances will be addressed. In the second part, I will describe policy-level intervention strategies which have been adopted in response to work stress in Taiwan and compare that with policy actions adopted in other East Asian countries. Unique features in terms of the nature of psychosocial work environment and social attitudes and reactions toward work stress from an international perspective will be explored.

\section{PREVENTION OF SLEEP DISORDERS AMONG SHIFT WORKERS AND DRIVERS}

Pierluigi Cocco. Department of Medical Sciences and Public Health, University of Cagliari, Cagliari, Italy

\subsection{6/oemed-2018-ICOHabstracts. 14}

Introduction Sleep is a vital function regulated by a circadian rhythm. Its restriction results in daytime sleepiness, which disrupts social life and affects behaviours that have survival value, particularly for occupations requiring a high level of alertness, such as shift workers and drivers.

Methods Data from published reports and unpublished preliminary results will be used to illustrate the genetics of the sleep/wake cycle and the mechanisms underlying the health consequences of sleep loss.

Result Shift-work can alter the sleep/wake cycle and the circadian rhythm of biological functions, which results in daytime sleepiness and disruption of social life. About 10\% shift workers complain daytime sleepiness or insomnia, impairment in their performance, and cardiovascular, digestive and neuropsychiatric symptoms. Polymorphisms in genes expressing the proteins that regulate the circadian functions result in different chronotypes with diverse capability of adapting to shift rotation schedules. Circadian genes also regulate the maintenance of energy balance; sleep loss is a contributor to the development of metabolic disorders, which min turn, are a major risk factor for obstructive sleep apnea syndrome (OSAS). Day time sleepiness is frequently consequent to OSAS, a major cause of deadly road accidents, and an occupational hazard for drivers of commercial and public transport vehicles and commuters to work, but also for the general public. Early detection of OSAS symptoms shall be part of health surveillance protocols of workers in commercial and public transport trades.

Discussion Several approaches are suggested to detect and monitor daytime sleepiness and OSAS among shift workers and long haul drivers, including specific questionnaires, and biomonitoring the salivary concentration of melatonin and cortisol level at a specific day time. A carefully designed biomonitoring protocol would help to reduce the health burden of sleep disorders and to save lives.

\section{EMERGENCY IN OCCUPATIONAL HEALTH: FROM PREPAREDNESS AND RESPONSE TO WELL-BEING?}

\footnotetext{
1,2,3 Alexis Descatha. ${ }^{1} A P-H P$, EMS (Samu92), Occupational Health Unit, University hospital of Poincare site, Garches, France; 'University Versailles St-Quentin, Versailles, France; ${ }^{3}$ Inserm, UMS 011 UMR1168, F-, Villejuif, France
}

10.1136/oemed-2018-ICOHabstracts. 15
Many occupational practitioners have to face emergencies in occupational setting, from life-threatening emergencies to current urgent care. Actually, workplace emergencies have singularities that usual emergency teams are not aware of - like use of hazardous substances, dangerous working conditions. Furthermore, responders and emergency professionals have to face to major hazards requiring prevention.

In the context of a new scientific committee created in ICOH in 2015 about Emergency Preparedness and Response in Occupational Health (EPROH), we aimed to take different examples to illustrate the importance for workers and population health and well-being, including emergency responders. Perspectives and challenges for next decades will be discussed.

\section{WORK STRESS, CAPITALISM AND THE IDEA OF PSYCHOSOCIAL SAFETY CLIMATE PSYCHOSOCIAL SAFETY CLIMATE: CAUSES AND COSTS OF PSYCHOSOCIAL RISKS AT WORK}

Maureen F Dollard. Asia Pacific Centre for Work Health and Safety, University of South Australia, A World Health Organisation Collaborating Centre in Occupational Health, Adelaide, Australia

\subsection{6/oemed-2018-ICOHabstracts. 16}

Introduction The driving beat of most modern societal structures is economic rationalism under capitalism. Relentless demands for increased profits, performance and productivity coupled with reduced resources, predispose workers to poor quality work conditions. In turn, poor quality work conditions can lead to mental and physical ill-health, with significant costs to organisations such as high rates of sickness absence and reduced performance, and costs to society such as loss of potential labour supply and high rates of unemployment. Organisations characterised by a good psychosocial safety climate (PSC) offer a point of resistance to these pressures.

Psychosocial safety climate concerns the value and priority given to worker psychological health compared to productivity imperatives likely achieved through economic rationalist approaches such as downsizing and lean structures. Far from undermining productivity we expect that pro-social options embodied in high PSC organisations that value worker psychological health will lead to better quality work options, increased meaningfulness, increased possibility for creativity and innovation, and reduced productivity costs associated with sickness absence and presenteeism. This presentation responds to a public health priority and a call from the OECD to prevent and manage mental ill-health and promote health and well-being by drawing attention to the connexion between work and mental health.

Methods Multilevel evidence from around the globe will be presented to show that PSC precedes work quality (demands, resources) and the social-relational aspects of work (harassment and bullying, social support). Evidence supporting the expansion of work stress theories to include national (e.g., culture, legislation and regulation, corrupt values, welfare regimes, union representation), organisational (e.g., PSC) and team level factors will be explored. International research showing the impact of PSC on working conditions, health and productivity; cost estimates for improving PSC at work; PSC benchmarks for job strain and depression; and implications for work systems improvements will be discussed. 